\title{
RESEARCH
}

Open Access

\section{Gut microbiota steroid sexual dimorphism and its impact on gonadal steroids: influences of obesity and menopausal status}

Jordi Mayneris-Perxachs ${ }^{1,2}$, María Arnoriaga-Rodríguez ${ }^{1,2}$, Diego Luque-Córdoba ${ }^{3,4}$, Feliciano Priego-Capote ${ }^{3,4}$, Vicente Pérez-Brocal ${ }^{5,6}$, Andrés Moya ${ }^{5,6,7}$, Aurelijus Burokas ${ }^{8,9}$, Rafael Maldonado ${ }^{8,10}$ and José-Manuel Fernández-Real ${ }^{1,2^{*}}$ (i)

\begin{abstract}
Background: Gonadal steroid hormones have been suggested as the underlying mechanism responsible for the sexual dimorphism observed in metabolic diseases. Animal studies have also evidenced a causal role of the gut microbiome and metabolic health. However, the role of sexual dimorphism in the gut microbiota and the potential role of the microbiome in influencing sex steroid hormones and shaping sexually dimorphic susceptibility to disease have been largely overlooked. Although there is some evidence of sex-specific differences in the gut microbiota diversity, composition, and functionality, the results are inconsistent. Importantly, most of these studies have not taken into account the gonadal steroid status. Therefore, we investigated the gut microbiome composition and functionality in relation to sex, menopausal status, and circulating sex steroids.

Results: No significant differences were found in alpha diversity indices among pre- and post-menopausal women and men, but beta diversity differed among groups. The gut microbiota from post-menopausal women was more similar to men than to pre-menopausal women. Metagenome functional analyses revealed no significant differences between post-menopausal women and men. Gonadal steroids were specifically associated with these differences. Hence, the gut microbiota of pre-menopausal women was more enriched in genes from the steroid biosynthesis and degradation pathways, with the former having the strongest fold change among all associated pathways. Microbial steroid pathways also had significant associations with the plasma levels of testosterone and progesterone. In addition, a specific microbiome signature was able to predict the circulating testosterone levels at baseline and after 1-year follow-up. In addition, this microbiome signature could be transmitted from humans to antibiotic-induced microbiome-depleted male mice, being able to predict donor's testosterone levels 4 weeks later, (Continued on next page)
\end{abstract}

\footnotetext{
* Correspondence: jmfreal@idibgi.org

'Department of Endocrinology, Diabetes and Nutrition, Departament de Ciències Mèdiques, Hospital of Girona "Dr JosepTrueta", Girona Biomedical Research Institute (IdibGi), University of Girona, Carretera de França s/n, 17007 Girona, Spain

${ }^{2}$ CIBERobn Pathophysiology of Obesity and Nutrition, Instituto de Salud Carlos III, Madrid, Spain

Full list of author information is available at the end of the article
} 
(Continued from previous page)

implying that the microbiota profile of the recipient mouse was influenced by the donor's gender. Finally, obesity eliminated most of the differences observed among non-obese pre-menopausal women, post-menopausal women, and men in the gut microbiota composition (Bray-Curtis and weighted unifrac beta diversity), functionality, and the gonadal steroid status.

Conclusions: The present findings evidence clear differences in the gut microbial composition and functionality between men and women, which is eliminated by both menopausal and obesity status. We also reveal a tight link between the gut microbiota composition and the circulating levels of gonadal steroids, particularly testosterone.

Keywords: Sex, Gender, Gonadal steroids, Testosterone, Progesterone, Microbiome, Sexual dimorphism

\section{Background}

The gut microbiota composition is known to be changed in parallel to a myriad of environmental factors, being diet and antibiotic/drug exposures the main determinants. There is some evidence that sex may influence the diversity, composition, and function of gut bacterial microbiota, although the results are inconsistent. Initial studies evaluating a relatively small number of subjects with limited technical capabilities, showed little or no significant differences attributed to gender. In a 2005 study on 91 subjects of northern European origin (France, Denmark, Germany, the Netherlands, and the $\mathrm{UK})$, there were no significant differences in gut microbiota between sexes according to principal component analysis [1]. A 2006 study, conducted in samples from France, Germany, Italy, and Sweden found higher levels of the Bacteroides-Prevotella group among males [2]. In a 2008 Chinese study using group-specific DGGE profiling of Bacteroides spp., a higher abundance of Bacteroides thetaiotaomicron was found among males [3]. More recent large population-wide studies also failed to show any major sex-specific differences in the gut microbiota diversity, complexity, or composition [4-6].

However, some other recent studies have highlighted the importance of sex as a factor affecting the human gut microbiota. Several studies have reported that men have lower microbial diversity than women [6-8]. In a large cohort study involving two independent extensively phenotyped cohorts, the Belgian Flemish Gut Flora Project $(n=1,106)$ and the Dutch LifeLines-DEEP study $(n$ $=1,135)$, sex had a 10th effect size among 69 factors that were shown to correlate significantly with overall microbiome community variation [7]. In another study, sex was also associated with the gut microbiota composition overall, with women having lower abundance of Bacteroidetes [9]. Comparisons at the species level in a Dutch cohort study reported sex associations with twelve microbial species (including Alistipes onderdonkii, Bilophila, Lachnospiraceae, and Bifidobacterium species increased in women) [8]. After multiple corrections, the relative abundance of only Akkermansia muciniphila was higher in females. Analysis of a healthy cohort of
300 individuals revealed that men were three times more likely than women to harbor stool community type with higher relative abundances of Ruminococcaceae, Faecalibacterium, and Alistipes, but lower Bacteroides and lack of Prevotella [10]. In one Japanese study, significantly higher levels of Prevotella, Megamonas, Fusobacterium, and Megasphaera were found among males, while Bifidobacterium, Ruminococcus, and Akkermansia were higher among females [11]. Ruminococcus was also more abundant among Chinese females compared with males [12]. However, the results of each study regarding the differences in microbial taxa between sexes are inconsistent.

Of note, the majority of studies evaluating sex differences in gut microbiota did not take into account the influence of menopausal status. Menopausal status is known to affect gut microbiome, with premenopausal women having a higher abundance of SCFA producing bacteria compared to postmenopausal women and agematched men [13, 14]. Specifically, the relative abundances of the SCFA producing genera Prevotella, Ruminococcus, and Roseburia were reported to depend both on sex and hormonal status. In a recent study involving three large cross-sectional cohorts, the association between sex and alpha diversity was much stronger in young adults than in middle-aged adults [15] and no differences were observed between men and women with a mean age of 60 years [16]. This suggests the menopause may play a role in the inconsistent results observed in all the previous studies.

Despite the role for female sex hormones in gastrointestinal transit $[17,18]$, clinical evidence for a change in gut microbiota composition according to circulating hormonal status is very limited in humans. Correlations with estradiol levels suggest positive associations with Gammaproteobacteria class and unknown family from Mixococcales (Proteobacteria-Lipopolysaccharide (LPS) producers), while bacterial family Prevotellaceae was negatively correlated [13]. Oral contraceptives and ovariectomy are also associated with changes in gut microbiota [8]. Importantly, castration in male mice abolishes sexual difference of gut microbiota composition, 
suggesting a critical role for testosterone in the complexity and diversity of virile males [19].

Given all these evidences, we hypothesized changes in gut microbiota composition and functionality according to sex and menopausal status. We also aimed to evaluate the potential influence of circulating gonadal steroids, as very few studies have studied these important confounding factors. We found that testosterone was the main factor associated with the gut microbiota signature. Finally, we explored whether this signature could be transmitted to mice using microbiota transplantation.

\section{Results}

Clinical characteristics of the study subjects at baseline $(n=131)$ and after 1-year follow-up $(n=81)$ are described in Table 1 and Supplementary Table 1 , respectively.

\section{Influences of gender and menopausal status on the gut microbiome composition}

We did not find significant differences in alpha diversity indices among pre-menopausal women, post-menopausal women, and men (Fig. 1a). However, Bray-Curtis and weighted unifrac beta diversity measures both indicated significant differences in the microbiome composition among groups (Fig. 1b). In particular, pre-menopausal women had different microbial composition compared to men based on either Bray-Curtis $(P=0.042)$ or weighted unifrac $(P=$ 0.044 ) beta diversity, whereas no differences were observed between the other groups. When subjects were studied according to the obesity status, no differences were found in any of the alpha diversity indices either in non-obese (Supplementary Figure 1a) or obese individuals (Supplementary Figure $1 \mathrm{~b}$ ), although in the former group, pre-menopausal tended to have higher alpha diversity than post-menopausal women and men based on the Chaol index. Bray-Curtis and weighted unifrac beta diversity measures revealed different microbiome compositions between pre-menopausal women and men $(P=0.008$ and $P=0.004$, respectively $)$ and post-menopausal women and men $(P=0.042$ and $P=$ 0.015 , respectively) in non-obese subjects (Supplementary Figure 1c,d). Remarkably, these differences were eliminated in obese subjects (Supplementary Figure 1e,f).

Differential abundance taxa between pre-menopausal women, post-menopausal women, and men were identified using DESeq2 analysis adjusting for age and obesity. We identified 273 taxa with significantly different read counts between pre-menopausal women and men (Fig. 1c, Supplementary Table 2). Compared to pre-menopausal women, a lower number of taxa differed from men (103) in post-menopausal women (Fig. 1d, Supplementary Table 3). A total of 90 taxa were differentially abundant between pre-

Table 1 Baseline characteristics of subjects according to the gender and menopausal status

\begin{tabular}{|c|c|c|c|c|}
\hline Clinical data & Pre-menopausal $(n=44)$ & Post-menopausal $(n=45)$ & Men $(n=42)$ & $P$ \\
\hline Age & $41.9[34.4-48.4]$ & $58.6[55.4-59.6]$ & $46.1[39.2-54.3]$ & $<0.001$ \\
\hline Polycystic ovary syndrome (\%) & $3(7 \%)$ & $0(0 \%)$ & $0(0 \%)$ & 0.05 \\
\hline Alcohol intake (g/day) & $0.0[0.0-2.1]$ & $0.86[0.0-2.5]$ & $2.3[0.0-12.3]$ & 0.003 \\
\hline Smoking (no, former, yes) (\%) & $63.6,27.3,9.1$ & $46.7,44.4,8.9$ & $51.9,35.9,12.2$ & 0.202 \\
\hline Obesity (\%) & 61.4 & 55.6 & 57.1 & 0.849 \\
\hline $\mathrm{BMI}\left(\mathrm{kg} / \mathrm{m}^{2}\right)$ & $39.5[25.0-44.3]$ & $32.8[24.4-41.4]$ & $34.1[27.5-45.1]$ & 0.304 \\
\hline Fat mass (kg) & $46.3[21.8-61.3]$ & 39.8 [22.6-50.3] & $34.5[23.5-61.1]$ & 0.530 \\
\hline $\mathrm{SBP}(\mathrm{mmHg})$ & $128.5(18.6)$ & $131.0(18.7)$ & $133.5(20.4)$ & 0.088 \\
\hline $\mathrm{DBP}(\mathrm{mmHg})$ & $73.5(12.8)$ & $76.5(9.3)$ & $76.0(11.8)$ & 0.276 \\
\hline HDL cholesterol (mg/dL) & $52.0[45.5-62.0]$ & $62.0[51.5-72.5]$ & $48.5[37.8-58.0]$ & $<0.001$ \\
\hline Triglycerides (mg/dL) & $79.5[58.7-106.7]$ & $96.0[72.5-132.0]$ & $104[67.5-162.7]$ & 0.071 \\
\hline Fasting plasma glucose (mg/dl) & 94.0 [88.0-98.8] & $96.0[90.5-104.5]$ & $98.0[90.8-104.0]$ & 0.197 \\
\hline HOMA-IR & $5.68[2.56-8.02]$ & $3.01[1.78-5.90]$ & $3.57[2.35-6.44]$ & 0.051 \\
\hline IVGTT (80-120 min) (mg/dL) & $106.7(8.18)$ & $103.1(8.69)$ & $103.5(9.16)$ & 0.162 \\
\hline M-clamp (mg/(kg.min)) & $5.30[2.50-10.4]$ & $6.86[4.60-10.3]$ & $5.01[2.61-8.39]$ & 0.137 \\
\hline $\mathrm{HbA} 1 \mathrm{c}(\%)$ & $5.50(0.29)$ & $5.56(0.32)$ & $5.52(0.29)$ & 0.606 \\
\hline hs-CRP (mg/dL) & 3.24 [0.91-9.49] & $2.53[0.64-6.10]$ & $1.69[0.74-3.14]$ & 0.065 \\
\hline
\end{tabular}

Results are expressed as number and frequencies for categorical data, mean and standard deviation for normal distributed continuous variables, and median and interquartile range for non-normal distributed continuous variables

Differences between groups were assessed using the $x^{2}$ test for categorical variables, one-way ANOVA for normal quantitative variables, and the Kruskal-Wallis test for non-normal quantitative variables

$B M I$ body mass index, DBP diastolic blood pressure, $H b 1 A c$ glycated hemoglobin, $H D L$ high density lipoprotein, $h s$-CRP high sensitive C-reactive protein, IVGTT intravenous glucose tolerance test, $S B P$ systolic blood pressure 


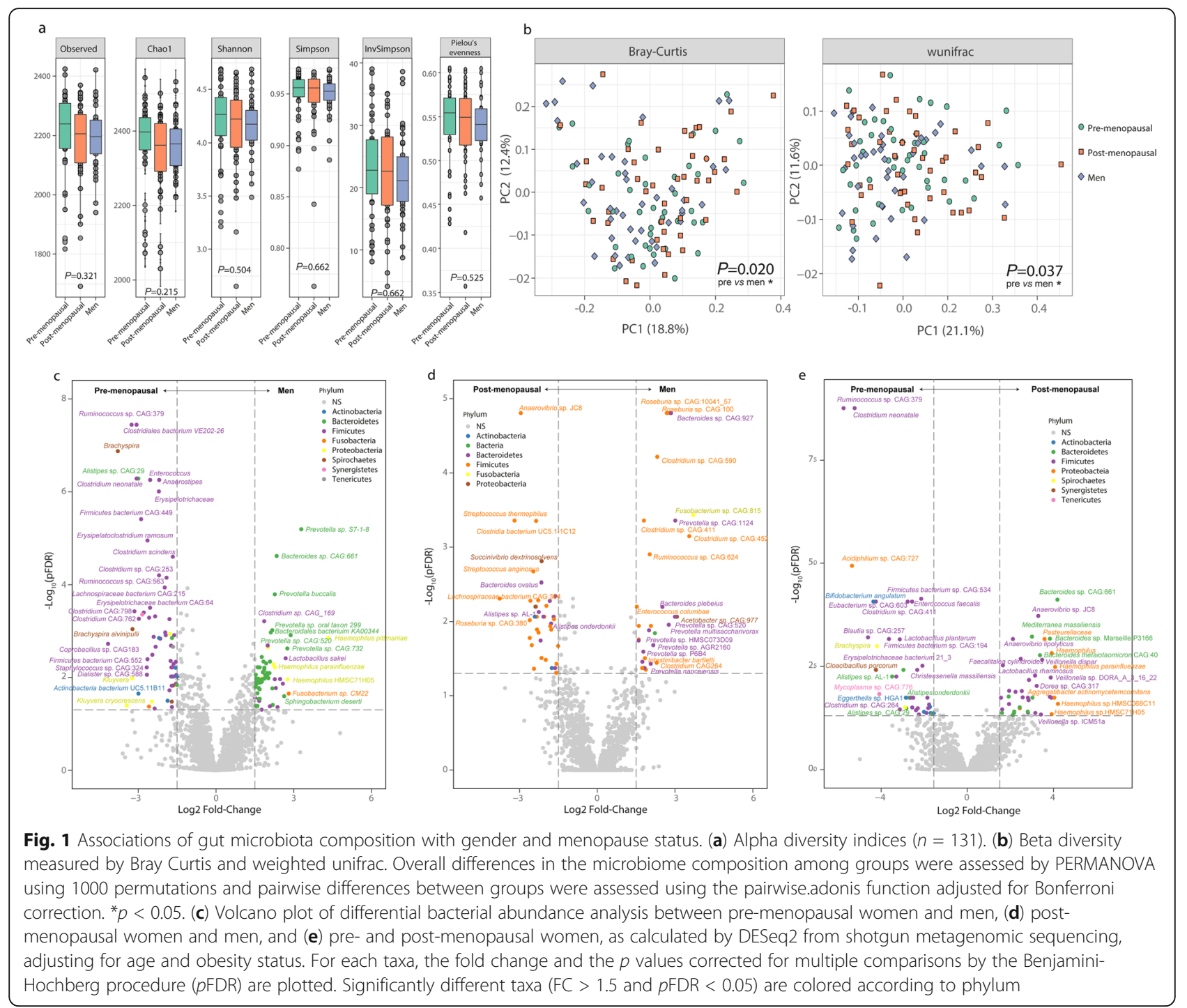

menopausal and post-menopausal women (Fig. 1e, Supplementary Table 4).

At the species level, men and post-menopausal women had higher abundances of several species belonging to the Pasteurellaceae (Haemophilussp. HMSC71H05, Haemophilus parainfluenzae), Bacteroidaceae (Bacteroides sp. CAG:661, Bacteroides stercoris CAG:120, Mediterranea massiliensis, Bacteroides pectinophilus CAG:437), Prevotellaceae (Prevotella sp. P6B1, Prevotella sp. CAG: 617), and Clostridiaceae families (Clostridium sp. CAG: 169, butyrate-producing bacterium SS3/4), but lower abundances of several species, particularly Ruminococcussp. CAG:379, Clostridium neonatale, Alistipessp. CAG:29, Cloacibacillus porcorum, Erysipelotrichaceae bacterium CAG:64, and Brachyspira, compared to premenopausal women.

Compared to pre- and post-menopausal women, men had higher abundances of several Prevotellaceae species
(Prevotella sp. CAG:520, Prevotella multisaccharivorax, Prevotella sp. AGR2160, Prevotella nanceiensis, Prevotella sp. HMSC073D09, Prevotella bergensis, Prevotella sp. CAG:924, Prevotella ruminicola) and Clostridium sp. CAG:277, but lower levels of Roseburia sp. CAG:380, Firmicutes bacterium CAG:884, Lachnospiraceae bacterium CAG:364, Bacillus sp. CAG:988, Succinivibrio dextrinosolvens, Clostridium sp. CAG:557, Bacteroides dorei CAG: 222, and Lachnospiraceae bacterium CAG:215.

At the family level, men had higher Pasteurellaceae, Cytophagaceae, Idiomarinaceae, Prevotellaceae, Sphingobacteriaceae, Flavobacteriaceae, and Fibrobacteriaceae, whereas the abundance of Synergistaceae, Fusobacteriaceae, Myxococcaceae, Actinobacteria, Enterococcaceae, and Christensenellaceae was higher in pre-menopausal women (Supplementary Figure 1a). Once again, men had higher abundances of Fibrobacteriaceae and Sphingobacteriaceae compared to post-menopausal women, 
whereas the latter had higher levels of Acetobacteriaceae, Leuconostocaceae, Acholeplasmataceae, Lactobacillaceae, Streptococcaceae, and Lentisphaerae (Supplementary Figure 1b). Post-menopausal women had higher abundances of Pasteurellaceae, Comamonadaceae, and Lentisphaerae, but lower abundances of Acholeplasmataceae, Enterococcaceae, Micrococcales, and Christensenellaceae than pre-menopausal women (Supplementary Figure 1c).

\section{Influence of gender and menopausal status differences on the gut microbiome functionality}

Metagenome functional analyses based on KEGG pathways revealed significant differences between premenopausal women and men (Fig. 2a). Remarkably, enrichment in steroid biosynthesis and degradation of metabolic pathways were found in the gut microbiota of premenopausal women compared to men. Other differentially expressed pathways included arginine biosynthesis and metabolism, purine and pyrimidine metabolism, one carbon pool by folate and carbohydrate metabolism pathways. Conversely, we did not find significant differences in the microbiome functionality between postmenopausal women and men, despite having different bacterial composition. Indeed, we only found an enrichment in arginine biosynthesis and metabolism pathways in postmenopausal women compared to men $\left(\log _{2}\right.$ Fold-change $=-1.33, p$ FDR $\left.=0.034\right)$, which was also increased in premenopausal women compared to men. In line with the functional differences between premenopausal women and men, the gut microbiota of premenopausal women was enriched in pyrimidine and one carbon pool by folate pathways compared to postmenopausal women (Fig. 2b). In addition, these differences were blunted by obesity. Therefore, while these functional differences between premenopausal women and men and between pre- and postmenopausal women were evident in non-obese individuals (Supplementary Figure 2a,b), no differences in the gut microbiota functionality were observed in obese subjects. Finally, most of the identified bacterial pathways had significant correlations with the plasma levels of gonadal steroids, particularly progesterone and testosterone (Fig. 2c). Interestingly, after adjustment for age, obesity, and menopause status, the steroid degradation pathway had a significant positive correlation with progesterone levels.

\section{Gender, menopausal status, and obesity differences in the gonadal steroids}

A significant O-PLS-DA model predicted the gender and menopause status from the levels of several steroids (Fig. 3a). Unsupervised analyses by principal component analysis also showed a clear between- group difference (Fig. 3b, c). Among steroids, estrone, estradiol, androstenedione, testosterone, DHT (Dihydrotestosterone), $\quad$ Progesterone, 17 $\alpha$ hydroxyprogesterone, and $17 \alpha$-hydroxypregnenolone have significant between-group differences (Fig. 3d-f). As expected, males had higher levels of testosterone, DHT, and 17 $\alpha$-hydroxyprogesterone compared to both preand post-menopausal women. Pre-menopausal women had higher estrone, estradiol, progesterone, and androstenedione compared to men, whereas post-menopausal women had lower estrone, androstenedione, and 17 $\alpha$ hydroxypregnenolone compared to men. The levels of estrone, estradiol, androstenedione, testosterone,

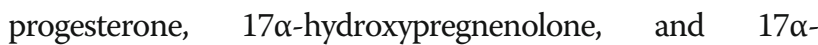
hydroxyprogesterone were higher in pre-menopausal compared to post-menopausal women. Remarkably, most of these differences were lost in obese subjects (Supplementary Figure 3). In particular, while lean pre-menopausal women had higher circulating levels of testosterone, DHEA (dehydroepiandrosterone), progesterone, $17 \alpha$ hydroxyprogesterone, and 17 $\alpha$-hydroxypregnenolone, compared to lean post-menopausal women, no significant differences were found in the levels of these steroids between obese pre- and post-menopausal women. In addition, estrogens levels were higher in obese post-menopausal women compared to lean post-menopausal women, whereas no differences were found between obese and lean premenopausal women. This is in agreement with the fact that pre-menopausal women mainly synthesize estrogens in the ovary, while in post-menopausal women ovarian biosynthesis is replaced by peripheral site synthesis, and in obese postmenopausal women, adipose tissue is the main source of estrogen biosynthesis.

\section{Gut microbial associations with gonadal steroids}

We then evaluated the possible associations between gut microbiota composition and the circulating concentrations of the main gonadal steroids. A significant O-PLS model was obtained for the prediction of testosterone (Fig. 4a, b) levels from microbiota families. In particular, Prevotellaceae, Cytophagaceae, Fibrobacteriaceae, Sphingobacteriaceae, and Idiomarinaceae were positively associated with testosterone levels, whereas several families from the Actinobacteria, Proteobacteria, Firmicutes, and Verrucomicrobia phylum were negatively associated with testosterone levels. We further evaluated the association between the gut microbiota families and the circulating testosterone levels using a negative binomial distribution by DESeq2 and adjusting for age and obesity (Fig. 4c). Again, Fibrobacteriaceae, Idiomarinaceae, and several families from the Bacteroidetes phylum (Sphingobacteriaceae, Cytophagaceae, Prevotellaceae, and Flavobacteriaceae) still had positive associations with testosterone levels, whereas Acholeplasmataceae, Verrucomicrobia, and several families 


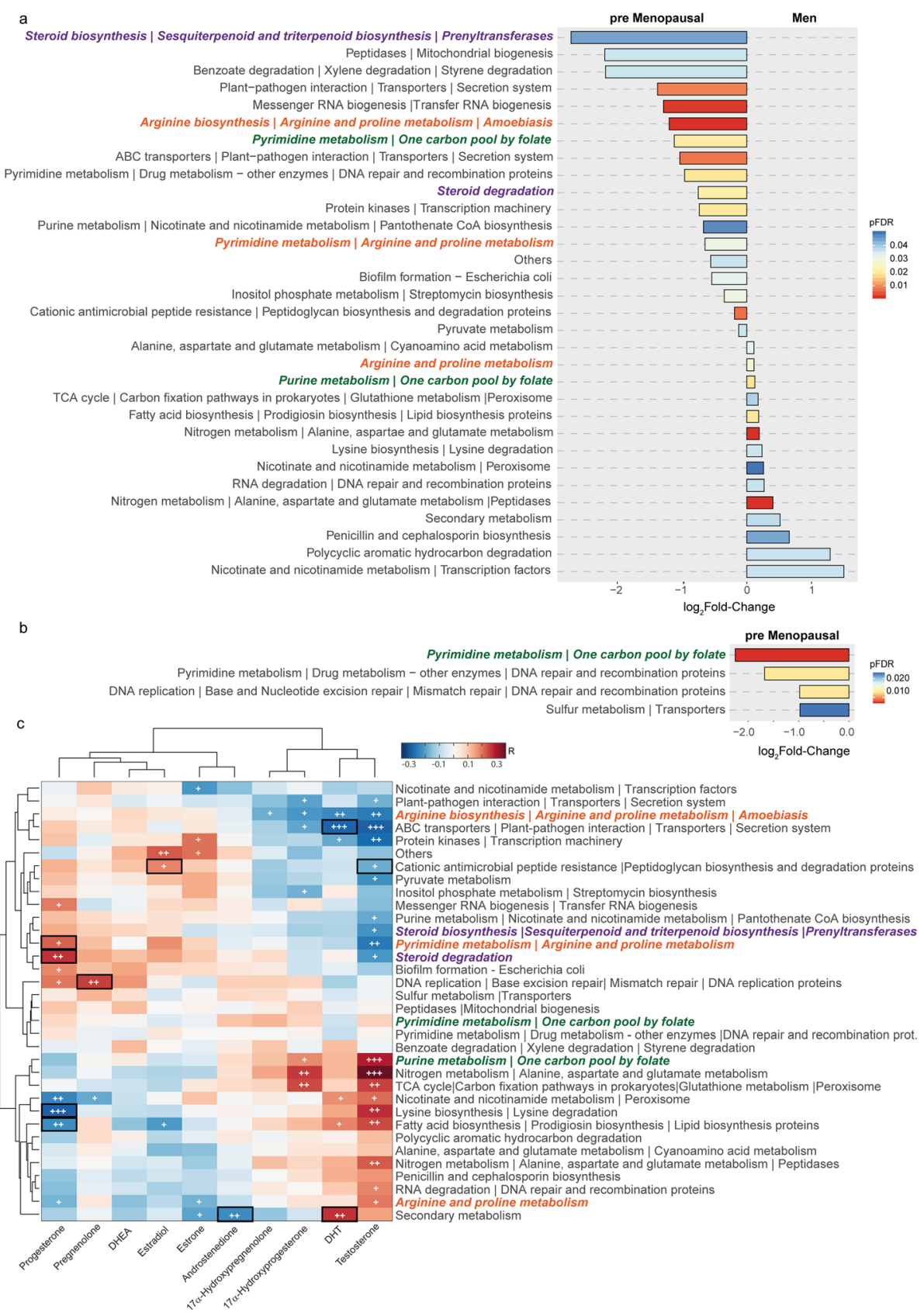

Fig. 2 Associations of gut microbiota functionality with gender and menopause status. (a) Fold change for the significant differential KEGG pathways between pre-menopausal women and men, and (b) pre- and post-menopausal women, identified by DESeq2 adjusting for age and obesity status. Bars are colored according to the Benjamini-Hochberg corrected $p$ values ( $p F D R$ ). (c) Spearman correlation heatmap among the abundance of identified KEGG pathways and plasma concentrations of gonadal steroids. Clustering was based on Euclidean distances and Ward linkage. Significance: ,$+<0.05 ;++,<0.01 ;+++,<0.001$. Significant associations after adjusting for age, obesity, and sex are highlighted with a black box

from the Proteobacteria (Gammaproteobacteria, Myxococcaeae, Xanthomonadacea), Firmicutes (Lactobacillaceae) and Actinobacteria phyla had the strongest negative fold change. We were also able to predict the circulating progesterone levels from the gut microbiota composition, although the predictive performance of the O-PLS model was lower $\left(Q^{2} Y=0.04, P=0.003\right)$.
When we considered the obesity status, better predictive models for the testosterone (Supplementary Figure 4a,b) and progesterone $\left(Q^{2} Y=0.11, P=0.008\right)$ levels were obtained in non-obese individuals, whereas the gut microbiota composition of obese subjects could not predict the circulating concentrations of these two gonadal steroids $\left(Q^{2} Y=-0.12\right.$ and $\left.Q^{2} Y=-0.25\right)$. Noteworthily, 

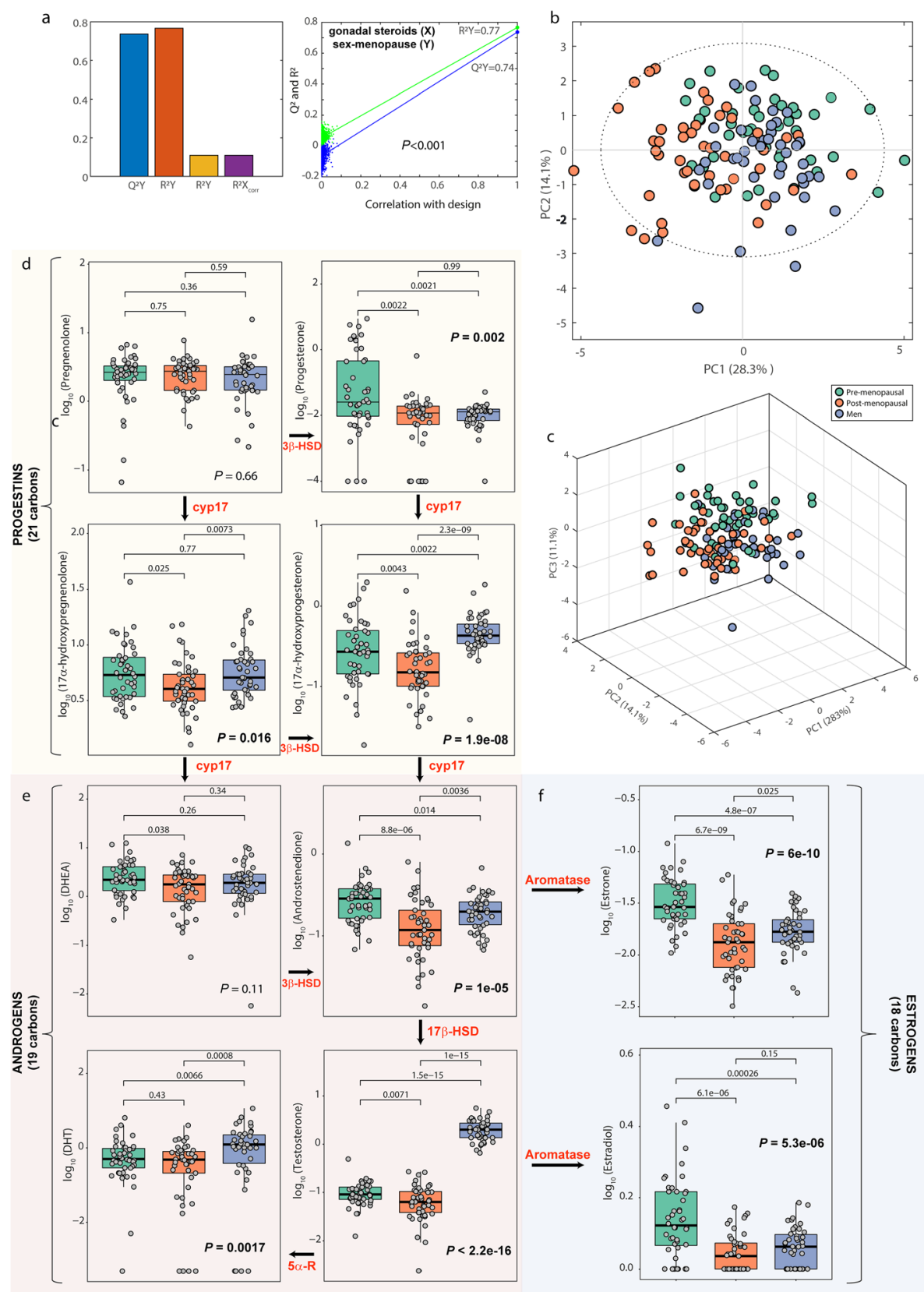

Fig. 3 Gender and menopausal status differences in gonadal steroids. (a) Goodnessoffit $\left(R^{2} Y\right)$, goodness of prediction $\left(Q^{2} Y\right)$, and permutation tests for the O-PLS-DA model predicting the sex and menopause status from the circulating gonadal steroid levels. (b, c) Principal component analysis score plots for the plasma levels of gonadal steroids colored according to the gender group. (d) Boxplots for the concentrations of progestin, (e) androgens, and (f) estrogens converted to base 10 logarithmic values. Differences among groups were analyzed by a Kruskal-Wallis test, and pairwise comparisons were assessed by Wilcoxon test. Significant differences are highlighted in bold

the same bacterial family signature was still positively associated with testosterone levels in non-obese subjects, whereas Verrucomicrobia and Akkermansiaceae (also from Verrucomicrobia phylum) were predictive of lower circulating testosterone concentrations.

The gut microbiota composition was also able to predict the plasma testosterone levels $(n=81) 1$ year later
(Supplementary Figure 4c). In particular, we identified a consistent microbial signature positively associated with testosterone concentrations that included Cytophagaceae, Prevotellaceae, Fibrobacteriaceae, Sphingobacteriaceae, Flavobacteriaceae, and Idiomarinaceae (Supplementary Figure 4d). Once again, several families from the Verrucomicrobia, including Optitutaceae, Puniceicoccaceae, and 

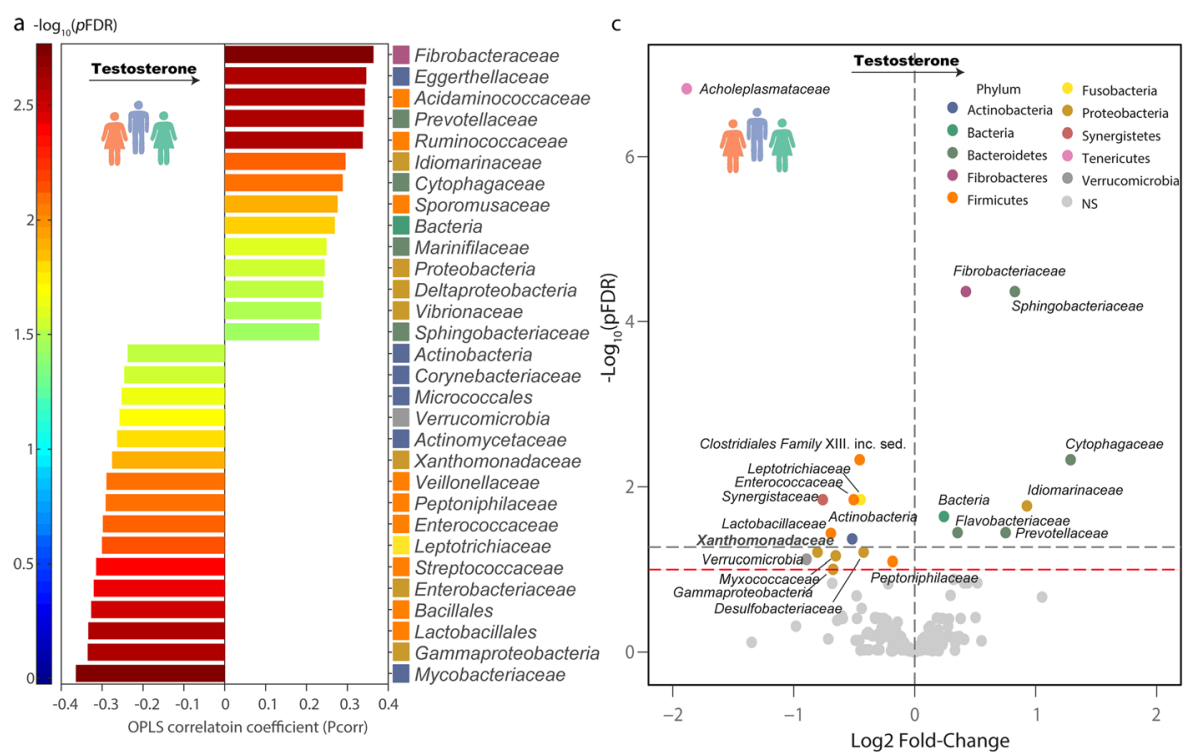

b
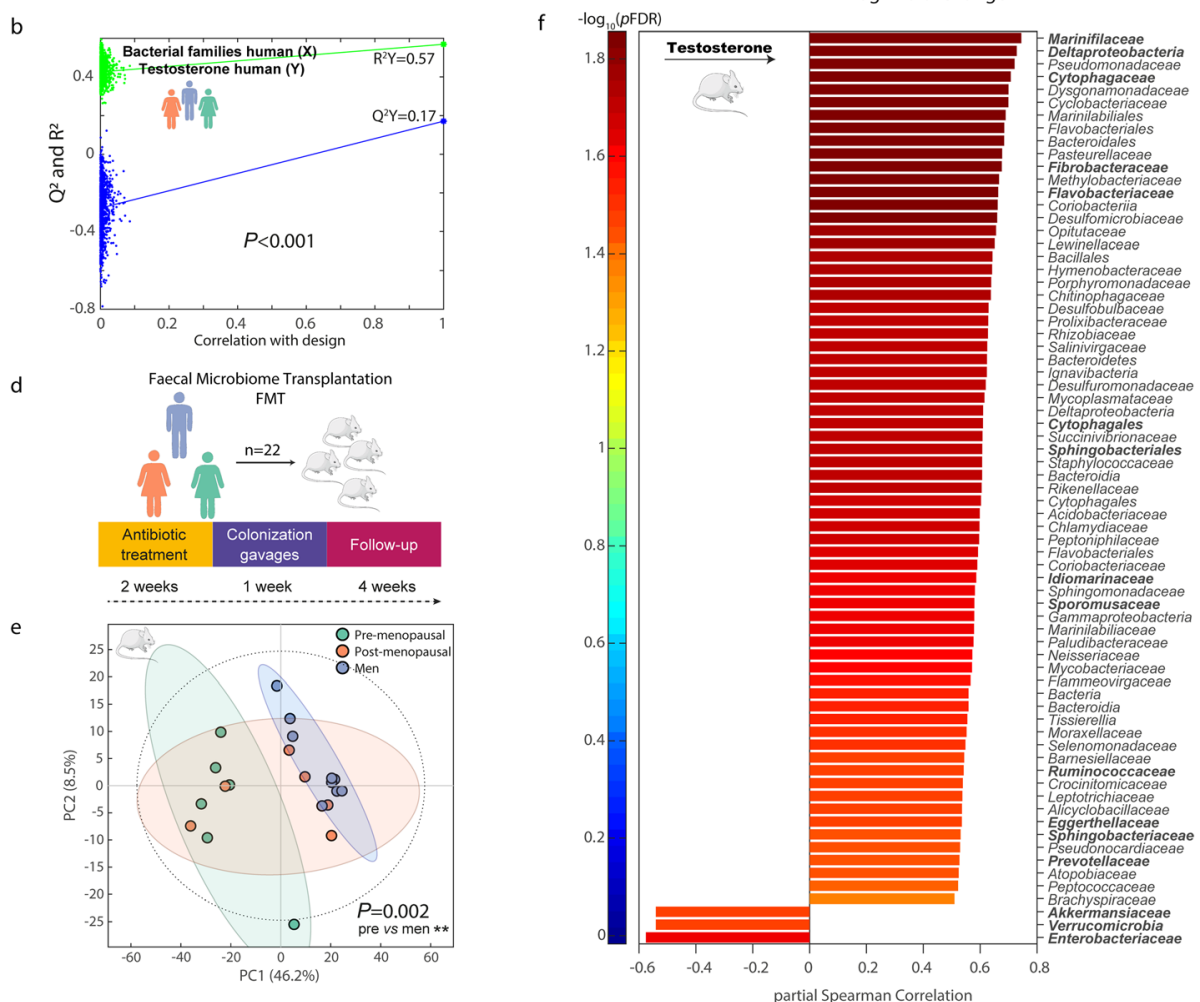

Fig. 4 Gut microbial associations with circulating testosterone concentrations. (a) Significant gut bacterial families predicting plasma testosterone levels in humans identified by O-PLS modeling. (b) Permutation tests for the goodness-of-fit $\left(R^{2} \gamma\right.$ ) and goodness of prediction $\left(Q^{2} \gamma\right)$ for the O-PLS model between bacterial families and circulating testosterone concentrations in humans. (c) Volcano plot of gut bacterial families associated with testosterone levels identified by DESeq2, adjusting for age and obesity status. For each family, the fold change and the Benjamini-Hochberg corrected $p$ values ( $p$ FDR) are plotted. Significant families (gray dashed line: $p F D R<0.05$; red dashed line: $p$ FDR $<0.1$ ) are colored according to phylum. (d) Experimental design for the fecal microbiota transplantation study in mice. Fecal samples from 22 human donors (11 men and 11 women) were transplanted to 22 mice after 2 weeks of antibiotic treatment. After 28 days of colonization gavage, mice fecal samples were collected and analyzed by shotgun metagenomic sequencing. (e) Principal component analysis score plot based on recipient's mice bacterial families colored according to human donor sex and menopause status. Overall differences in the microbiome composition were assessed by PERMANOVA using 1000 permutations and Euclidean distances. Pairwise differences between groups were assessed using the pairwise.adonis function adjusted for Bonferroni correction. ${ }^{* *} p<0.01$. (f) Significant recipient's mice bacterial families predicting human donor circulating testosterone levels identified by partial Spearman correlation adjusted by age and obesity status 
an unknown family of Verrucomicrobia, have negative associations with testosterone.

\section{The sex-related signature is transmitted to mice through the gut microbiota}

We finally evaluated the hypothesis that the sex-related signature could be transplanted to mice through the gut microbiota. We transplanted the microbiota from 11 men and 11 women (age and obesity matched) to 22 male mice (Fig. 4d). Fecal microbiota transplantation from human donor to recipient mice resulted in a clear difference in the microbiome composition of male mice that received microbiota from pre-menopausal women and those that transplanted with microbiota from male donors 28 days after transplantation $(P=0.006)$ (Fig. 4e). Remarkably, most mice receiving microbiota from obese post-menopausal women had a microbiota profile similar to those that received microbiota from male donors (Fig. 4e).

Interestingly, when evaluating the mice gut microbiota composition 28 days later under a chow diet, we could successfully predict the donor testosterone and progesterone levels from the recipient's mice microbiota by O-PLS modeling (Supplementary Figure 4e, f). After partial Spearman correlation adjusted for donor's age and obesity status, several bacterial families predicting testosterone levels were the same in mice and humans (Fig. 4f). Specifically, Sphingobacteriaceae, Fibrobacteriaceae, Prevotellaceae, Cytophagaceae, Idiomarinaceae, Flavobacteriaceae, Eggerthellaceae, Ruminococcaceae, Marinifilaceae, Deltaproteobacteria, and Sporomusaceae were positively associated with testosterone in both mice and humans, whereas Verrucomicrobia and Enterobacteriaceae had negative associations. Akkermansiaceae was also negatively associated with human donor testosterone in mice and non-obese subjects.

\section{Discussion}

Sexual dimorphism has long been associated with health and disease [20]. For example, men are at higher risk of CVD than premenopausal women, but this cardioprotection is lost after menopause [21], suggesting the contribution of gonadal steroids on susceptibility to disease. More recently, the gut microbiota has also been implicated on the etiology of several diseases [22-24]. Therefore, the gut microbiota may be the driving force underlying the observed sexual dimorphism in disease susceptibility.

There is some evidence to support that the gut microbiota differs between sex, although the results are inconsistent $[1-10,12,15]$, partly because sexual dimorphism in the gut microbiome may also be influenced by diet, age, obesity, ethnicity, and genotype [6]. The findings of the current study confirm some of the previous observations, with higher abundances of Prevotella and Fusobacterium species in males, and of Alistipes, and Lachnospiraceae bacterium species in females. In addition, non-obese women also had higher abundances of several Akkermansia species compared to non-obese men. Importantly, the majority of the studies did not consider menopausal status as a potential confounding factor. Interestingly, we found that the number of species from genera differentiating men and women was lower after menopause. We also found that premenopausal women had higher abundances of several Alistipes, Bifidobacterium, and Ruminococcus species but lower abundances of Bacteroides, Prevotella, and Haemophilus species compared to postmenopausal women and men. Therefore, our results suggest a masculinization of the gut microbiota composition after menopause.

This androgenization of the microbiome was further corroborated by functional analyses. Therefore, while we found several differentially abundant bacterial pathways between premenopausal women and men, only the arginine biosynthesis and metabolism pathway differed between the postmenopausal and men microbiome. This pathway was enriched in women independently of menopausal status compared to men. It is one of the central pathways for the biosynthesis of arginine, a precursor of nitric oxide (NO), which plays a key role in the regulation of blood pressure. In fact, Forte et al. showed that the whole-body production of NO is higher in premenopausal women than in men, although these authors could not decipher the cellular origin of NO [25]. This could partly explain the substantial sex differences observed in hypertension from epidemiological studies [23]. Consistently, alterations in the arginine and proline metabolism have been reported in individuals at high risk of CVD [26] and spontaneously hypertensive rats [27]. We also found a consistent enrichment in the onecarbon pool by folate pathway in the microbiome of premenopausal women compared to post-menopausal women and men. Folic acid is the most important determinant of homocysteine levels, whose levels have been related to CVD [28]. Notably, men and post-menopausal women have higher homocysteine levels and are at higher risk of CVD compared to pre-menopausal women $[29,30]$. Interestingly, we found that pre-menopausal women had higher abundances of Lactobacillus plantarum compared to post-menopausal women and men. Unlike other Lactobacillus species, L. plantarum is unique in its capacity to synthesize folate [31].

On the other hand, a limited number of studies have shown that the sex difference in the gut microbiota is influenced by the grade of obesity [12, 16]. Accordingly, we found significant differences in the gut microbiota functionality between non-obese premenopausal women 
and men, but no differences were observed in obese subjects. In addition, none of these studies have taken into account the menopausal status. Similarly, we found that the functional differences in the gut microbiota between pre- and post-menopausal women disappeared in obese women. Therefore, obesity has the effect of erasing the sexual dimorphism associated with the microbiome. Low levels of several plasma steroids, including testosterone and 17-hydroxyprogesterone, have been associated with higher adiposity and visceral fat [32]. These two steroid hormones were precisely among those that did differ between non-obese pre- and post-menopausal women, but not between obese individuals. Due to the relationship between gonadal steroids and the gut microbiome, it is possible that a decreased production of steroid hormones in obese subjects partly explains the lack of sexual dimorphism in the gut microbiota of these individuals.

\section{Associations of the gut microbiota with gonadal steroids}

There is evidence that some bacterial species are able to metabolize and catabolize estrogen and androgens and their precursors, thereby affecting their systemic levels [33-35]. For example, bacteria possess several enzymes involved in the steroid biosynthesis and metabolism such as hydroxysteroid dehydrogenase (HSD). Notably, we found that the gut microbiota of pre-menopausal women was more enriched in steroid biosynthesis and degradation pathways and these microbial pathways had significant associations with the plasmatic levels of testosterone and progesterone. HSD is abundant in species of the Actinobacteria phylum, specifically in Bifidobacterium species [36], and Proteobacteria and Firmicutes phyla [37]. Interestingly, we found that premenopausal women had higher abundances of Actinobacteria species, and in particular several Bifidobacterium species, compared to men. In addition, we found that several families from the Actinobacteria, Proteobacteria, and Firmicutes phyla were predictive of lower testosterone circulating concentrations.

In line with the ability of the gut microbiota to modulate steroid levels, animal models demonstrate that the microbiome is essential in maintaining regular estrogen cycles, testosterone levels, and reproductive roles in both sexes. Germ-free non-obese diabetic male mice had lower testosterone concentrations than conventional mice [38]. In addition, removal of the microbiota increased the circulating testosterone concentration in female mice but decreased the levels in male mice [38]. The negative associations between the relative abundance of some bacterial species and serum testosterone levels are in line with its removal leading to increased circulating testosterone in females.
In turn, the microbiome may be affected by hormone levels. Previous investigations have focused on the influence of estrogen on microbiota composition [39-45]. For example, the relative abundance of Bacteroidetes was significantly increased in an ovariectomized rat model [46]. In another study, normal females had significantly lower Proteobacteria abundance and Firmicutes to Bacteroidetes ratio, but higher Bifidobacterium to Enterobacteriaceae ratio and increased Akkermansia abundance, compared to normal male and ovariectomized (OVX) females [45]. Some of these observations were recapitulated with 17- $\beta$-estradiol treatments in males and OVX females. Treatment with progesterone in OVX mice resulted in a significant change in the gut microbiota composition, with particular increases in Lactobacillus species [47]. In women, the relative abundance of the Gammaproteobacteria class and an unknown family from Myxococcales correlated positively with estradiol levels, while Prevotellaceae had a negative correlation [13]. Consistently, we found that Prevotellaceae family was positively associated with testosterone levels, whereas Gammaproteobacteria, Lactobacillaceae, and Myxococcaceae had negative associations.

Post-menopausal women did not exhibit higher levels of testosterone than pre-menopausal women suggesting that a mechanism other than androgenization may be also responsible for the differences in gut microbiota between pre-menopausal women vs. post-menopausal women and men.

\section{Effects of testosterone}

Only a few studies reported the associations between the gut microbiota and testosterone. Prenatal exposure to testosterone cypionate in female rats resulted in a lower abundance of Akkermansia, Bacteroides, Lactobacillus, and Clostridium in adult female offspring [48]. Gonadectomy along with a high fat diet was associated with increased genera of Ruminococcaceae family in male mice of three different strains and reduced Akkermansia genus in female mice [49]. However, males on a chow diet had higher Ruminococacceae than females. In a recent investigation using 16S rRNA sequencing methods, the authors observed significant positive correlations between the relative abundance of bacteria in the Actinobacter, Dorea, Ruminococcus, and Megamonas genera and serum testosterone concentrations in men [50]. An indirect way to consider the effects of testosterone levels is through women or animal models of polycystic ovary syndrome (PCOS), which is characterized by an excessive production of androgens or testosterone. In female rats, the increase in testosterone after Letrozole-induced PCOS led to decreased Lactobacillus, Ruminococcus, and Clostridium, but higher Prevotella [51]. Letrozole treatment of adult mice also resulted in a higher relative 
abundance of genera from Lachnospiraceae, Ruminococcaceae, and Peptococcaceae, and lower Lactobacillus [52]. In a recent human study, Bacteroidetes, Firmicutes, and Verrucomicrobia phyla differed between women with PCOS and controls, with obesity having a driving role in the development of dysbiotic microbiota [53]. In particular, women with PCOS had higher relative abundances of several Ruminococcus spp. In line with these results, in the current study, the abundance of some Lachnospiraceae bacterium (Firmicutes) and Ruminococcus spp. (Firmicutes) was higher in males vs. premenopausal females, whereas several Prevotella spp. (Bacteroidetes) were higher in males compared to women. We also found that non-obese women also had higher abundances of several Akkermansia spp. (Verrucomicrobia) compared to non-obese men. O-PLS modeling also revealed a negative association between Verrucomicrobia and Akkermansiaceae family and testosterone levels in non-obese individuals. However, the most consistent association with testosterone in O-PLS models were with Prevotellaceae, Cytophagaceae, Ruminococcaceae, Fibrobacteriaceae, Sphingobacteriaceae, and Idiomarinaceae. Remarkably, this microbial signature predictive of the testosterone levels could be transmitted to mice by faecal microbiota transplantation (FMT), being this signature in mice able to predict donor's serum testosterone 28 days later. However, we must point out the one limitation of the present FMT study is that only male mice were used.

Although the number was small, women with PCOS did not have elevated testosterone levels when compared with the rest of premenopausal women and did not significantly influence the findings concerning testosterone levels. The median testosterone levels in women with PCOS were 0.0785 [0.0531-0.1165], in the range of that observed among premenopausal women and with no outliers. These women with PCOS had also low androstenedione levels compared to the other pre-menopausal women $(0.1057$, 0.1431 , and 0.3008 in the range from $0.069-1.35$ observed for androstenedione in pre-menopausal women). Similar results were observed for DHEA and DHT. In addition, we performed the KEGG metagenome functional analyses again removing these three pre-menopausal women and the results were almost the same.

\section{Conclusions}

In conclusion, our results evidence a clear difference in the gut microbial composition and functionality between men and women, which is influenced by both menopausal and obesity status. Therefore, menopause is proposed to induce an androgenization of the microbiome, whereas obesity overrides the sex and menopause differences observed in non-obese individuals. The gut microbiota composition was tightly linked to the circulating levels of gonadal steroids, particularly testosterone.

\section{Methods \\ Clinical study (cross-sectional) \\ Recruitment of study subjects}

From January 2016 to October 2017, a cross-sectional case-control study was undertaken in the Endocrinology Department of JosepTrueta University Hospital. We included consecutive subjects with obesity (body mass index, BMI $\geq 30 \mathrm{~kg} / \mathrm{m}^{2}$ ) and age- and sex-matched nonobese subjects $\left(18.5<\mathrm{BMI}<30 \mathrm{~kg} / \mathrm{m}^{2}\right)$, with an age range of 27.2-66.6 years $(n=131)$. Exclusion criteria were the following: type 2 diabetes mellitus, chronic inflammatory systemic diseases, acute or chronic infections in the previous month; use of antibiotic, antifungal, antiviral, or treatment with proton-pump inhibitors; severe disorders of eating behavior or major psychiatric antecedents; and excessive alcohol intake $(\geq 40 \mathrm{~g} \mathrm{OH} /$ day in women or $80 \mathrm{~g} \mathrm{OH} /$ day in men). The institutional review board-Ethics Committee and the Committee for Clinical Research (CEIC) of Dr.JosepTrueta University Hospital (Girona, Spain) approved the study protocol, and informed written consent was obtained from all participants.

\section{Clinical and laboratory parameters}

Body composition was assessed using a dual energy Xray absorptiometry (DEXA, GE Lunar, Madison, Wisconsin).

\section{Longitudinal cohort}

A total of $n=81$ subjects were followed for 1 year and plasma samples were collected after 1 year of follow-up for the determination of steroids.

\section{Extraction of fecal genomic DNA and whole-genome shotgun sequencing}

Total DNA was extracted from frozen human stools using the QIAamp DNA mini stool kit (Qiagen, Courtaboeuf, France). Quantification of DNA was performed with a Qubit 3.0 fluorometer using the QubitTM dsDNA HS assay kit (Thermo Fisher Scientific, Carlsbd, CA, USA), and $1 \mathrm{ng}$ of each sample $(0.2 \mathrm{ng} / \mu \mathrm{l})$ was used for shotgun library preparation for high-throughput sequencing, using the Nextera DNA Flex Library Prep kit (Illumina, Inc., San Diego, CA, USA) according to the manufacturers' protocol. Sequencing was carried out on a NextSeq 500 sequencing system (Illumina) with $2 \times$ 150 -bp paired-end chemistry (120Gb/120 millions of reads), at the facilities of the Sequencing and Bioinformatic Service of the FISABIO (Valencia, Spain). 
Metagenome sequences were trimmed and quality controlled using the PRINSEQ-lite-0.20.4 program [54], and forward and reverse reads were joined using FLAS H-1.2.11 [55], applying a minimum overlap of 10, maximum overlap of 150, and maximum mismatch density of 0.1 . Host reads were removed by mapping against the host reference genome, by using bowtie2-2.3.4.3 [56] with end-to-end and very sensitive options. For functional analyses an assembly of the host-free reads into contigs was implemented by MEGAHIT v1.1.2 [57], followed by a mapping of those reads against the contigs with bowtie2, appending the reads that did not assemble to the contigs. Next, the program Prodigal v2.6.3 [58] was used for predicting open reading frames (orfs). The functional annotation was carried out with HMMER [59] against the Kyoto Encyclopedia of Genes and Genomes (KEGG) database [60] to obtain the functional at three levels, subcategory, pathway, and annotation of the genes. The filtering of the best annotations and the assignment of the orf annotation to every read were carried out using the statistical package $R$ 3.6.0 [61], which also was used to count the aligned reads and to add the category and its coverage, and finally to build abundance matrices.

In parallel, taxonomic annotation was implemented with Kaiju v1.6.2 [62] on the concatenated host-free reads, and the resulting file was split by sample. Addition of lineage information, lineage parsing for undetermined ranks, counting of taxa, and generation of the operational taxonomic unit (OTU) absolute and relative abundance matrices for all samples were performed using the package $\mathrm{R}$.

\section{Full automated method for determination of steroids}

The determination of steroids in serum was carried out by liquid chromatography with tandem mass spectrometry (LC-MS/MS) detection. Sample preparation involved solid-phase extraction (SPE) in an automated unit from Spark Holland (Emmen, The Netherlands), which was on-line coupled to a LC-MS/MS with a triple quadrupole mass detector from Agilent (Palo Alto, USA). A volume of $175 \mu \mathrm{L}$ of serum is introduced in an opaque vial with a glass insert and located in the autosampler of the SPE system. The online SPE protocol consists of a first equilibration step of the sorbent cartridge (C18 HySphere, Spark-Holland, $10 \mathrm{~mm}$ length and $2 \mathrm{~mm}$ diameter, $8 \mu \mathrm{m}$ particle size), with $10 \mathrm{~mL}$ of methanol $(\mathrm{MeOH})$ followed by two solvation steps with $1 \mathrm{~mL}$ of $10 \%(\mathrm{v} / \mathrm{v})$ acetonitrile $(\mathrm{ACN})$ in water containing $0.1 \%(\mathrm{v} / \mathrm{v})$ of formic acid. Meanwhile, the sample loop is filled with $100 \mu \mathrm{L}$ of sample with a subsequent load in the sorbent cartridge with $2 \mathrm{~mL}$ of $10 \%(\mathrm{v} / \mathrm{v}) \mathrm{ACN}$ in water containing $0.1 \%(\mathrm{v} / \mathrm{v})$ of formic acid. The loading step leads to the consequent retention of the analytes in the sorbent and the removal of sample matrix components to waste. Then, the sorbent is rinsed with $1 \mathrm{~mL}$ of $20 \%(\mathrm{v} / \mathrm{v}) \mathrm{ACN}$ in water and, then, the analytes are eluted with the chromatographic mobile phase (for 9 min and $30 \mathrm{~s}$ elution time).

Chromatographic separation of steroids was carried out by a Kinetex $\mathrm{C} 18$ analytical column (particle size $2.6 \mu \mathrm{m}, 10 \mathrm{~cm}$ length, and $3 \mathrm{~mm}$ inner diameter) from Phenomenex (Torrance, CA, USA). A security guard C18 cartridge ( $4 \mathrm{~mm}$ length and $3 \mathrm{~mm}$ inner diameter) from Phenomenex was used to preserve the integrity of the analytical column. The initial chromatographic mobile phase was composed of $40 \%(\mathrm{v} / \mathrm{v}) \mathrm{MeOH}$ in water containing $0.2 \mathrm{mM} \mathrm{NH} 4 \mathrm{~F}$ as an ionizing agent. A first gradient was applied for $3.5 \mathrm{~min}$ up to $68 \%$ of $\mathrm{MeOH}$, a second gradient for $6 \mathrm{~min}$ up to $71 \%$ of $\mathrm{MeOH}$, followed by a third gradient for $4 \mathrm{~min}$ to $80 \%$ of $\mathrm{MeOH}$ and, finally, a last gradient for $1 \mathrm{~min}$ up to $100 \%$ of $\mathrm{MeOH}$. The temperature of the chromatographic column was set at $35^{\circ} \mathrm{C}$ and the flow rate is $300 \mu \mathrm{L}$ min- 1 .

The MS/MS detection was carried out in multiple reaction monitoring (MRM) with electrospray ionization (ESI) in fast switching polarity mode. The capillary voltage was set at $+/-3.5 \mathrm{kV}$ and the nozzle voltage at 0.5 $\mathrm{kV}$. The nebulizer pressure was set at $45 \mathrm{psi}$ and the flow of N2 as drying gas at $12 \mathrm{~L} \mathrm{~min}-1$ and $325^{\circ} \mathrm{C}$. Parameters for MRM detection are listed in Supplementary Table 5. Calibration models were prepared by analysis of aliquots of a serum pool spiked with variable concentrations of the target steroids. Endogenous content of each steroid in the sample loop was subtracted in the preparation of the calibration models. Isotopically labeled steroids were used as internal standards for quantitative analysis of structurally similar analytes.

\section{Animal procedures}

Eight-weeks-old male C57BL/6 J mice (Charles River, France), weighing 20-26g at the beginning of the experiment were used in this study. Mice were housed individually in controlled laboratory conditions with the temperature maintained at $21 \pm 1{ }^{\circ} \mathrm{C}$ and humidity at 55 $\pm 10 \%$ during all the study. Animal procedures were conducted in strict accordance with the guidelines of the European Communities Directive 86/609/EEC regulating animal research and were approved by the local ethical committee (CEEA-PRBB). All the experiments were performed under blinded conditions.

Then, mice were given a cocktail of ampicillin and metronidazole, vancomycin (all at $500 \mathrm{mg} / \mathrm{L}$ ), ciprofloxacin $\mathrm{HCl}(200 \mathrm{mg} / \mathrm{L})$, imipenem $(250 \mathrm{mg} / \mathrm{L})$ once daily for 14 consecutive days in drinking water, as previously described [63]. Seventy-two hours later, animals were colonized via daily oral gavage of donor microbiota $(150 \mu \mathrm{L})$ for 3 days. Animals were orally gavaged with fecal 
material from age- and obesity-matched healthy volunteers ( $n=22,11$ men and 11 women). To offset potential confounder and/or cage effects and to reinforce the donor microbiota phenotype, booster inoculations were given twice per week throughout the study. Animals were maintained on normal mouse chow diet (rat and mouse no. 1 maintenance diet, Special Diet Services ${ }^{\circ}$ ) and water ad libitum, and were weighed every week. Food intake was the same in the two groups. At the end of the study, the animals were consecutively sacrificed. The cecum was removed, weighed and stored, and the feces collected and stored at $-80^{\circ} \mathrm{C}$ for further microbiota analysis. Fecal microbiota composition from mice was analyzed following the same procedures as for humans.

\section{Statistical analysis}

Normal distribution and homogeneity of variances in clinical variables were tested using the Shapiro-Wilk and Levene's tests. Results are expressed as number and frequencies for categorical variables, mean and standard deviation for normal distributed continuous variables, and median and interquartile range for non-normal distributed continuous variables. Differences between study groups were assessed using the $\chi^{2}$ for categorical variables, one-way ANOVA for normal quantitative variables, and Kruskal-Wallis tests for non-normal quantitative variables. These analyses were performed using SPSS version 19 (SPSS, Inc, Chicago, IL).

For metagenomics data, taxa were first filtered so that only those with more than 10 reads in at least two samples were selected. Then, differential bacterial taxa or KEGG pathways between groups were identified with the $R$ package DESeq2 [64]. It uses a generalized linear model of counts based on a negative binomial distribution, scaled by a normalization factor that accounts for differences in sequencing depth between samples. Significance testing was then assessed using the Wald test and the $p$ values corrected for multiple comparisons by the Benjamini-Hochberg procedure ( $p$ FDR) [65]. In all models, age and obesity status were included as covariates.

Predications of circulating gonadal steroid levels from bacterial families were performed using orthogonal partial least squares (O-PLS) models with unit variance using in-house MATLAB scripts. The abundances of bacterial families after applying a variance stabilizing transformation using DESeq2 were used as the descriptor matrices $(\mathrm{X})$ to predict the testosterone levels as the response $(\mathrm{Y})$. The predictive performance $\left(Q^{2} Y\right)$ of each model was calculated using a tenfold cross-validation approach, and model validity was established by permutation testing (1000 permutations). The significance of O-
PLS correlation coefficients was adjusted by the Benjamini-Hochberg method ( $p$ FDR).

\section{Supplementary information}

Supplementary information accompanies this paper at https://doi.org/10. 1186/s40168-020-00913-x.

Additional file 1: Supplementary Table 1. Clinical characteristics of subjects after 1-year follow-up according to the gender and menopausal status.

Additional file 2: Supplementary Table 2. DESeq2 results for the differential expressed bacterial taxa between pre-menopausal women and men.

Additional file 3: Supplementary Table 3. DESeq2 results for the differential expressed bacterial taxa between post-menopausal women and men.

Additional file 4: Supplementary Table 4. DESeq2 results for the differential expressed bacterial taxa between pre-menopausal women and post-menopausal women.

Additional file 5: Supplementary Table 5. MRM parameters for determination of steroids and isotopically labelled standards by LC-MS/ MS.

Additional file 6: Supplementary Figure 1. Associations of gut microbiota composition in non-obese and obese subjects and bacterial families with gender and menopause status. Alpha diversity indices in a) non-obese and b) obese individuals. Beta diversity in non-obese subjects measured by c)Bray-Curtis and d) weighted unifrac. Beta diversity in obese subjects measured by e) Bray-Curtis and f) weighted unifrac. Overall differences in the microbiome composition among groups were assessed by PERMANOVA using 1000 permutations and pairwise differences between groups were assessed using the pairwise.adonis function adjusted for Bonferroni correction. ${ }^{*}, P<0.05$; $\left.{ }^{* *}, P<0.01 .9\right)$ Volcano plot of differential bacterial families abundance analysisbetween premenopausal women and men, h) post-menopausal women and men, and i) pre- and post-menopausal women, as identified by DESeq2 from shotgun metagenomic sequencing data, adjusting for age and obesity status. For each family, the fold change and the $p$ values corrected for multiple comparisons by the Benjamini-Hochberg procedure ( $p F D R$ ) are plotted. Significantly different taxa ( FC > 1 and pFDR $<0.05)$ are coloured according to phylum.

Additional file 7: Supplementary Figure 2. Associations of gut microbiota functionality with gender and menopause status in nonobese subjects. a) Fold change for the significant differential KEGG pathways between pre-menopausal women and men, and b) pre- and postmenopausal women, identified by DESeq2 adjusting for age and obesity status. Bars are colored according to the Benjamini-Hochberg corrected $p$ values ( $p F D R)$.

Additional file 8: Supplementary Figure 3. Gender and menopausal status differences in gonadal steroids according to the obesity status. Boxplots for the concentrations of progestin,androgens, and estrogens converted to base 10 logarithmic values. Differences among groups were analyzed by a Kruskal-Wallis test, and pair-wise comparisons were assessed by the Wilcoxon test. Significant differences are highlighted in bold italics.

Additional file 9: Supplementary Figure 4. Gut microbial associations with circulating testosterone concentrations. a) Permutation tests for the goodness-of-fit $\left(R^{2} \gamma\right)$ and goodness of prediction $\left(Q^{2} \gamma\right)$ for the O-PLS model predicting plasma testosterone levels from bacterial families in non-obese individuals. b) Significant gut bacterial families identified by O-PLS modeling. c) Permutation tests for the goodness-of-fit $\left(R^{2} \gamma\right)$ and goodness of prediction $\left(Q^{2} Y\right.$ for the O-PLS model predicting plasma testosterone levels after 1-year follow-up from bacterial families at baseline in humans. d) Significant gut bacterial families identified by O-PLS modeling. e) Permutation tests for the goodness-of-fit $\left(R^{2} Y\right)$ and goodness of prediction $\left(Q^{2} Y\right)$ for the O-PLS model predicting human donor circulating 
testosterone and $\mathbf{f}$ ) progesterone concentrations from recipient's mice bacterial families.

\section{Acknowledgements}

We are in debt with the subjects involved in this project. We also thank Emili LosHuertos and Oscar Rovira for their help in the recruitment of the subjects.

\section{Authors' contributions}

J.M.P. researched the data, performed the statistical analysis, and wrote the manuscript. M.A.-R. researched the data and wrote the manuscript. D.L.-C. and F.P.-C performed the analysis of gonadal steroids. V.P-B and A.M. contributed with the determination and analysis of the microbiota. A.B. and R.M. performed the experiments in mice. J.M.F.-R. carried out the conception and coordination of the study and wrote the manuscript. All authors participated in the final approval of the version to be published. J.M.F.-R. is the guarantor of this work and, as such, had full access to all the data in the study and takes responsibility for the integrity of the data.

\section{Funding}

This work was partially supported by research grants FIS (PI15/01934) from the Instituto de Salud Carlos III from Spain, SAF2015-65878-R and AEISAF2017-84060-R from Ministry of Economy and Competitiveness, Prometeo/ 2018/A/133 from Generalitat Valenciana, Spain, and also by European Commission (FP7, NeuroPain \#2013-602891; \#H2020-SC1-2019-2-RTD-848099 (PAINFACT)), the Catalan Government (AGAUR, \#SGR2017-669, ICREA Academia Award 2015), the Spanish Instituto de Salud Carlos III (RTA, \#RD16/ 0017/0020), the Fondo Europeo de Desarrollo Regional (FEDER) through the Programa Interreg V-A España-Francia-Andorra (POCTEFA 2014-2020), and the European Regional Development Fund (project No. 01.2.2-LMT-K-718-020014) under grant agreement with the Research Council of Lithuania (LMTLT). María Arnoriaga Rodríguez is funded by a predoctoral Río Hortega contract (CM19/00190, co-funded by European Social Fund "Investing in your future") from the Instituto de Salud Carlos III, Spain. Jordi Mayneris-Perxachs is funded by the Miguel Servet Program from the Instituto de Salud Carlos III (ISCIII CP18/00009), co-funded by the European Social Fund "Investing in your future".

\section{Availability of data and materials}

The datasets used and/or analyzed during the current study are available from the corresponding author on reasonable request.

\section{Ethics approval and consent to participate}

Human study: The institutional review board-Ethics Committee and the Committee for Clinical Research (CEIC) of Dr. Josep Trueta University Hospital (Girona, Spain) approved the study protocol, and informed written consent was obtained from all participants.

Mice experiment: Animal procedures were conducted in strict accordance with the guidelines of the European Communities Directive 86/609/EEC regulating animal research and were approved by the local ethical committee (CEEA-PRBB)

\section{Consent for publication}

Not applicable.

\section{Competing interests}

The authors declare no competing interests.

\section{Author details}

${ }^{1}$ Department of Endocrinology, Diabetes and Nutrition, Departament de Ciències Mèdiques, Hospital of Girona "Dr JosepTrueta", Girona Biomedical Research Institute (IdibGi), University of Girona, Carretera de França s/n, 17007 Girona, Spain. ${ }^{2}$ CIBERobn Pathophysiology of Obesity and Nutrition, Instituto de Salud Carlos III, Madrid, Spain. ${ }^{3}$ Maimónides Institute of Biomedical Research (IMIBIC), Reina Sofía University Hospital, University of Cordoba, Cordoba, Spain. ${ }^{4}$ CIBERfes Frailty and Healthy Aging, Instituto de Salud Carlos III, Madrid, Spain. ${ }^{5}$ Department of Genomics and Health, Foundation for the Promotion of Health and Biomedical Research of Valencia Region (FISABIO-Public Health), Valencia, Spain. ${ }^{6} \mathrm{CIBER}$ in Epidemiology and Public Health (CIBEResp), Madrid, Spain. ${ }^{7}$ Institute for Integrative Systems Biology (I2SysBio), The University of Valencia and The Spanish National
Research Council (CSIC-UVEG), Valencia, Spain. ${ }^{8}$ Laboratory of Neuropharmacology, Department of Experimental and Health Sciences, Universitat Pompeu Fabra, Barcelona, Spain. ${ }^{9}$ Present address: Institute of Biochemistry, Life Sciences Center, Vilnius University, Vilnius, Lithuania.

${ }^{10}$ Hospital del Mar Medical Research Institute (IMIM), Barcelona, Spain.

Received: 3 July 2020 Accepted: 24 August 2020

Published online: 20 September 2020

\section{References}

1. Lay C, Rigottier-Gois L, Holmstrøm K, Rajilic M, Vaughan EE, De Vos WM, et al. Colonic microbiota signatures across five northern European countries. Appl Environ Microbiol. 2005:71:4153-5.

2. Mueller S, Saunier K, Hanisch C, Norin E, Alm L, Midtvedt T, et al. Differences in fecal microbiota in different European study populations in relation to age, gender, and country: a cross-sectional study. Appl Environ Microbiol. 2006;72:1027-33.

3. Li M, Wang B, Zhang M, Rantalainen M, Wang S, Zhou H, et al. Symbiotic gut microbes modulate human metabolic phenotypes. Proc Natl Acad Sci U S A. 2008:105:2117-22

4. Human Microbiome Project Consortium T. Structure, function and diversity of the healthy human microbiome the human microbiome project consortium*. Nature. 2012;486:207-14.

5. Odamaki T, Kato K, Sugahara H, Hashikura N, Takahashi S, Xiao J-Z, et al. Age-related changes in gut microbiota composition from newborn to centenarian: a cross-sectional study. BMC Microbiol. 2016;16:90.

6. Yatsunenko T, Rey FE, Manary MJ, Trehan I, Dominguez-Bello MG, Contreras $M$, et al. Human gut microbiome viewed across age and geography. Nature. 2012:486:222-7.

7. Falony G, Joossens M, Vieira-Silva S, Wang J, Darzi Y, Faust K, et al. Populationlevel analysis of gut microbiome variation. Science (80-). 2016;352:560-4.

8. Sinha T, Vich Vila A, Garmaeva S, Jankipersadsing SA, Imhann F, Collij V, et al. Analysis of 1135 gut metagenomes identifies sex-specific resistome profiles. Gut Microbes. 2019;10:358-66.

9. Dominianni C, Sinha R, Goedert JJ, Pei Z, Yang L, Hayes RB, et al. Sex, body mass index, and dietary fiber intake influence the human gut microbiome. PLoS One. 2015;10:e0124599.

10. Ding T, Schloss PD. Dynamics and associations of microbial community types across the human body. Nature. 2014;509:357-60.

11. Takagi T, Naito Y, Inoue R, Kashiwagi S, Uchiyama K, Mizushima K, et al. Differences in gut microbiota associated with age, sex, and stool consistency in healthy Japanese subjects. J Gastroenterol. 2019;54:53-63.

12. Gao X, Zhang M, Xue J, Huang J, Zhuang R, Zhou X, et al. Body mass index differences in the gut microbiota are gender specific. Front Microbiol. 2018; 9:1250.

13. Santos-Marcos JA, Rangel-Zuñiga OA, Jimenez-Lucena R, Quintana-Navarro GM, Garcia-Carpintero S, Malagon MM, et al. Influence of gender and menopausal status on gut microbiota. Maturitas. 2018:116:43-53.

14. Santos-Marcos JA, Haro C, Vega-Rojas A, Alcala-Diaz JF, Molina-Abril H, Leon-Acuña A, et al. Sex differences in the gut microbiota as potential determinants of gender predisposition to disease. Mol Nutr Food Res. 2019; 63:e1800870.

15. de la Cuesta-Zuluaga J, Kelley ST, Chen Y, Escobar JS, Mueller NT, Ley RE, et al. Age- and sex-dependent patterns of gut microbial diversity in human adults. mSystems; 2019;4.

16. Haro C, Rangel-Zúñiga OA, Alcalá-Díaz JF, Gómez-Delgado F, Pérez-Martínez $P$, Delgado-Lista J, et al. Intestinal microbiota is influenced by gender and body mass index. PLoS One. 2016;11:e0154090.

17. Jung HK, Kim DY, Moon $\mathrm{H}$. Effects of gender and menstrual cycle on colonic transit time in healthy subjects. Korean J Intern Med. 2003;18:181-6.

18. Wald A, Van Thiel DH, Hoechstetter L, Gavaler JS, Egler KM, Verm R, et al. Gastrointestinal transit: the effect of the menstrual cycle. Gastroenterology. 1981;80:1497-500

19. Yurkovetskiy L, Burrows M, Khan AA, Graham L, Volchkov P, Becker L, et al. Gender bias in autoimmunity is influenced by microbiota. Immunity. 2013; 39:400-12.

20. Gerdts E, Regitz-Zagrosek V. Sex differences in cardiometabolic disorders. Nat. Med. 2019;25:1657-66

21. Virani SS, Alonso A, Benjamin EJ, Bittencourt MS, Callaway CW, Carson AP, et al. Heart disease and stroke statistics -2020 update. Circulation. 2020;141: e139-e596. 
22. Cross TWL, Kasahara K, Rey FE. Sexual dimorphism of cardiometabolic dysfunction: gut microbiome in the play? Mol Metab. 2018;15:70-81.

23. Beale AL, Kaye DM, Marques FZ. The role of the gut microbiome in sex differences in arterial pressure. Biol Sex Differ. 2019;10:22.

24. Razavi AC, Potts KS, Kelly TN, Bazzano LA. Sex, gut microbiome, and cardiovascular disease risk. Biol. Sex Differ. 2019;10:29.

25. Forte P, Kneale BJ, Milne E, Chowienczyk PJ, Johnston A, Benjamin N, et al. Evidence for a difference in nitric oxide biosynthesis between healthy women and men. Hypertension. 1998;32:730-4.

26. Mao B, Yi Y, Mo Q, Yang C, Zhong Q. Metabolic profiling reveals the heterogeneity of vascular endothelial function phenotypes in individuals at extreme cardiovascular risk. RSC Adv. Royal Society of Chemistry. 2019;9: 30033-44.

27. Wang H, Wang X, Qi D, Sun M, Hou Q, Li Y, et al. Establishment of the circadian metabolic phenotype strategy in spontaneously hypertensive rats: a dynamic metabolomics study. J Transl Med. 2020;18:38.

28. Wald DS, Morris JK, Law M, Wald NJ. Folic acid, homocysteine, and cardiovascular disease: judging causality in the face of inconclusive trial evidence. Br Med J. 2006;333:1114-7.

29. Henry OR, Benghuzzi H, Taylor HA, Tucci M, Butler K, Jones L. Suppression of homocysteine levels by vitamin B12 and folates: age and gender dependency in the Jackson heart study. Am J Med Sci. 2012;344:110-5.

30. Gambacciani M, Mannella P. Homocysteine, menopause and cardiovascular disease. Menopause Int. 2007;13:23-6.

31. Sybesma W, Starrenburg M, Tijsseling L, Hoefnagel MHN, Hugenholtz J. Effects of cultivation conditions on folate production by lactic acid bacteria. Appl Environ Microbiol. 2003;69:4542-8.

32. He Z, Rankinen T, Leon AS, Skinner JS, Tchernof A, Bouchard C. Plasma steroids, body composition, and fat distribution: effects of age, sex, and exercise training. Int J Obes. 2018;42:1366-77.

33. Kwa M, Plottel CS, Blaser MJ, Adams S. The intestinal microbiome and estrogen receptor-positive female breast cancer. J Natl Cancer Inst. 2016; 108:djw029.

34. Ridlon JM, Ikegawa S, Alves JMP, Zhou B, Kobayashi A, lida T, et al. Clostridium scindens: a human gut microbe with a high potential to convert glucocorticoids into androgens. J Lipid Res. 2013;54:2437-49.

35. Lombardi P, Goldin B, Boutin E, Gorbach SL. Metabolism of androgens and estrogens by human fecal microorganisms. J Steroid Biochem. 1978;9:795801.

36. Nuriel-Ohayon M, Neuman H, Ziv O, Belogolovski A, Barsheshet $Y$, Bloch N, et al. Progesterone increases Bifidobacterium relative abundance during late pregnancy. Cell Rep. 2019;27:730-6 e3.

37. Kisiela M, Skarka A, Ebert B, Maser E. Hydroxysteroid dehydrogenases (HSDs) in bacteria - a bioinformatic perspective. J Steroid Biochem Mol Biol. 2012; 129:31-46.

38. JGM M, Frank DN, Mortin-Toth S, Robertson CE, Feazel LM, Rolle-Kampczyk $U$, et al. Sex differences in the gut microbiome drive hormone-dependent regulation of autoimmunity. Science (80- ). 2013:339:1084-8.

39. Flores R, Shi J, Fuhrman B, Xu X, Veenstra TD, Gail MH, et al. Fecal microbial determinants of fecal and systemic estrogens and estrogen metabolites: a cross-sectional study. J Transl Med. 2012;10:253.

40. Menon R, Watson SE, Thomas LN, Allred CD, Dabney A, Azcarate-Peril MA, et al. Diet complexity and estrogen receptor $\beta$ status affect the composition of the murine intestinal microbiota. Appl Environ Microbiol. 2013;79:5763-73.

41. Fuhrman BJ, Feigelson HS, Flores R, Gail MH, Xu X, Ravel J, et al. Associations of the fecal microbiome with urinary estrogens and estrogen metabolites in postmenopausal women. J Clin Endocrinol Metab. 2014;99: 4632-40.

42. Baker JM, Al-Nakkash L, Herbst-Kralovetz MM. Estrogen-gut microbiome axis: physiological and clinical implications. Maturitas. 2017;103:45-53.

43. Lindheim L, Bashir M, Münzker J, Trummer C, Zachhuber V, Leber B, et al. Alterations in gut microbiome composition and barrier function are associated with reproductive and metabolic defects in women with polycystic ovary syndrome (PCOS): a pilot study. PLoS One. 2017;12: e0168390.

44. Park S, Kim DS, Kang ES, Bin KD, Kang S. Low-dose brain estrogen prevents menopausal syndrome while maintaining the diversity of the gut microbiomes in estrogen-deficient rats. Am J Physiol Endocrinol Metab. 2018;315:E99-109.
45. Kaliannan K, Robertson RC, Murphy K, Stanton C, Kang C, Wang B, et al. Estrogen-mediated gut microbiome alterations influence sexual dimorphism in metabolic syndrome in mice. Microbiome. 2018;6:205.

46. Cox-York KA, Sheflin AM, Foster MT, Gentile CL, Kahl A, Koch LG, et al. Ovariectomy results in differential shifts in gut microbiota in low versus high aerobic capacity rats. Physiol Rep. 2015;3:e12488.

47. Sovijit WN, Sovijit WE, Pu S, Usuda K, Inoue R, Watanabe G, et al. Ovarian progesterone suppresses depression and anxiety-like behaviors by increasing the Lactobacillus population of gut microbiota in ovariectomized mice. Neurosci Res. 2019;S0168-0102(19)30142-7.

48. Sherman SB, Sarsour N, Salehi M, Schroering A, Mell B, Joe B, et al. Prenatal androgen exposure causes hypertension and gut microbiota dysbiosis. Gut Microbes. 2018;9:400-21.

49. Org E, Mehrabian M, Parks BW, Shipkova P, Liu X, Drake TA, et al. Sex differences and hormonal effects on gut microbiota composition in mice. Gut Microbes. 2016;7:313-22.

50. Shin J-H, Park Y-H, Sim M, Kim S-A, Joung H, Shin D-M. Serum level of sex steroid hormone is associated with diversity and profiles of human gut microbiome. Res Microbiol. 2019;170:192-201.

51. Guo Y, Qi Y, Yang X, Zhao L, Wen S, Liu Y, et al. Association between polycystic ovary syndrome and gut microbiota. PLoS One. 2016;11: e0153196.

52. Torres PJ, Skarra DV, Ho BS, Sau L, Anvar AR, Kelley ST, et al. Letrozole treatment of adult female mice results in a similar reproductive phenotype but distinct changes in metabolism and the gut microbiome compared to pubertal mice. BMC Microbiol. 2019;19:57.

53. Liang Y, Ming Q, Liang J, Zhang Y, Zhang H, Shen T. Gut microbiota dysbiosis in polycystic ovary syndrome (PCOS): association with obesity - a preliminary report. Can J Physiol Pharmacol.; 2020;cjpp-2019-0413.

54. Schmieder R, Edwards R. prinseq. Bioinformatics. 2011;27:863-4

55. Magoč T, Salzberg SL. FLASH: Fast length adjustment of short reads to improve genome assemblies. Bioinformatics. 2011;27:2957-63.

56. Langmead B, Salzberg SL. Fast gapped-read alignment with Bowtie 2. Nat Methods. 2012;9:357-9.

57. Li D, Liu CM, Luo R, Sadakane K, Lam TW. MEGAHIT: An ultra-fast singlenode solution for large and complex metagenomics assembly via succinct de Bruijn graph. Bioinformatics. 2015;31:1674-6.

58. Hyatt D, Chen GL, LoCascio PF, Land ML, Larimer FW, Hauser LJ. Prodigal: Prokaryotic gene recognition and translation initiation site identification. BMC Bioinformatics. 2010;11:119.

59. Durbin R, Eddy SR, Krogh A, Mitchison G. Biological sequence analysis: probabilistic models of proteins and nucleic acids. Press CU, editor. Cambridge, UK; 1998.

60. Kanehisa M. KEGG: Kyoto encyclopedia of genes and genomes. Nucleic Acids Res. 2000;28:27-30.

61. R Development Core Team. R: A language and environment for statistical computing. Computing RF for S, editor. Vienna, Austria.; 2013.

62. Menzel P, Ng KL, Krogh A. Fast and sensitive taxonomic classification for metagenomics with Kaiju. Nat Commun. 2016;7:11257.

63. Kelly JR, Borre Y, O' Brien C, Patterson E, El Aidy S, Deane J, et al. Transferring the blues: depression-associated gut microbiota induces neurobehavioural changes in the rat. J Psychiatr Res; 2016;82:109-118.

64. Love Ml, Huber W, Anders S. Moderated estimation of fold change and dispersion for RNA-seq data with DESeq2. Genome Biol; 2014;15:550.

65. Benjamini $Y$, Hochberg $Y$. Controlling the false discovery rate: a practical and powerful approach to multiple testing. J R Stat Soc Ser B (Methodological). 1995;57:289-300.

\section{Publisher's Note}

Springer Nature remains neutral with regard to jurisdictional claims in published maps and institutional affiliations. 